\title{
Amino-terminal arginylation as a degradation signal for selective autophagy
}

\author{
Hyunjoo Cha-Molstad ${ }^{1}$, Yong Tae Kwon ${ }^{2, *}$ E Bo Yeon Kim ${ }^{1, *}$ \\ ${ }^{1}$ World Class Institute, Korea Research Institute of Bioscience and Biotechnology, Cheongwon 28116, Korea, ${ }^{2}$ Protein Metabolism Medical \\ Research Center and Department of Biomedical Sciences, College of Medicine, Seoul National University, Seoul 03080, Korea
}

The ubiquitin-proteasome system and the autophagy lysosome system are the two major protein degradation machineries in eukaryotic cells. These two systems coordinate the removal of unwanted intracellular materials, but the mechanism by which they achieve this synchronization is largely unknown. The ubiquitination of substrates serves as a universal degradation signal for both systems. Our study revealed that the amino-terminal Arg, a canonical $\mathrm{N}$-degron in the ubiquitin-proteasome system, also acts as a degradation signal in autophagy. We showed that many ER residents, such as BiP, contain evolutionally conserved arginylation permissive pro- $\mathrm{N}$-degrons, and that certain inducers like dsDNA or proteasome inhibitors cause their translocation into the cytoplasm where they bind misfolded proteins and undergo amino-terminal arginylation by arginyl transferase 1 (ATE1). The amino-terminal Arg of BiP binds p62, which triggers p62 oligomerization and enhances p62-LC3 interaction, thereby stimulating autophagic delivery and degradation of misfolded proteins, promoting cell survival. This study reveals a novel ubiquitin-independent mechanism for the selective autophagy pathway, and provides an insight into how these two major protein degradation pathways communicate in cells to dispose the unwanted proteins. [BMB Reports 2015; 48(9): 487-488]

*Corresponding authors. Yong Tae Kwon, E-mail: yok5@snu.ac.kr; Bo Yeon Kim, E-mail: bykim@kribb.re.kr

http://dx.doi.org/10.5483/BMBRep.2015.48.9.176

Received 24 August 2015

Keywords: ATE1, p62, Autophagy

Abbreviations: ER, Endoplasmic reticulum; BiP, Binding immunoglobulin protein; GRP78, Glucose regulated protein78; HSPA5, Heat shock $70 \mathrm{kDa}$ protein 5; LC3, Microtubule-associated protein light chaain 3; LIR, LC3 interacting region; RIP1, Receptor interacting protein 1

Perspective to: Hyunjoo Cha-Molstad et al. (2015), N-terminal Arginilylation Targets Endoplasmic Reticulum Chaperone BiP to Autophagy Through p62 Binding, Nature Cell Biology, 17(7), 917-929, doi: 10.1038/ncb3177.
The ubiquitin-proteasome system (UPS) and the (macro) autophagy (hereafter autophagy) employ substrate ubiquitination as a common degradation signal. In UPS, ubiquitinated substrates are directly recruited to proteasomes via the docking of ubiquitin to the 195 regulatory subunit, while selective autophagy requires cargo receptors that specifically recognize ubiquitinated targets delivering them to prephagosomal structures (PAS). p62, also called sqstm1, is one of these critical cargo receptors, whose function has been implicated in the autophagic degradation of protein aggregates, damaged mitochondria and invading bacteria. p62 contains a LIR motif that binds the autophagosome marker LC3 and LC3-like proteins, and a carboxy terminal UBA domain that binds ubiquitin. It also contains an amino-terminal PB1 domain which is responsible for the homo- and hetero-oligomerization of p62, followed by a ZZ domain that is known to bind RIP1. Our recent report revealed that the ZZ domain of p62 also binds to the N-terminal arginine of arginylated BiP (also known as HSPA5 and GRP78). $\mathrm{BiP}$ is an ER chaperone that is cotranslationally translocated into the ER lumen, where its amino terminal ER signal peptide is cleaved by signal peptidase exposing a new evolutionally conserved amino-terminal glutamic acid at position 19, which is an arginylation permissive secondary $\mathrm{N}$-degron in the $\mathrm{N}$-end rule pathway. Based on our study, E19-BiP remains unarginylated inside ER since there is no ATE1 present, but certain cellular stresses, such as cytoplasmic dsDNA and inhibition of proteasomes, trigger the luminal $\mathrm{BiP}$ to retrotranslocate into the cytoplasm, where it undergoes post-translational arginylation by arginyl t-RNA-protein transferase 1 (ATE1). Our molecular and cellular biology data indicate that in the cytoplasm, BiP binds to misfolded proteins and the ZZ domain of p62 through its amino-terminal Arg.

p62 oligomerization is emerging as an important regulatory step of p62 activity and has been shown to be required for p62 targeting to PAS. In addition, p62 interaction with LC3 is required for the p62 integration into maturing autophagosomes. Our data also indicates that this binding of the amino-terminal Arg of BiP to the ZZ domain of p62 leads to p62 oligomerization and aggregation, which stimulates the delivery of Arg-BiP-misfolded protein aggregates to PAS, and their integration into maturing autophagosomes by enhancing the p62 interaction with LC3. Arg-BiP-misfolded protein aggregates eventually undergo 


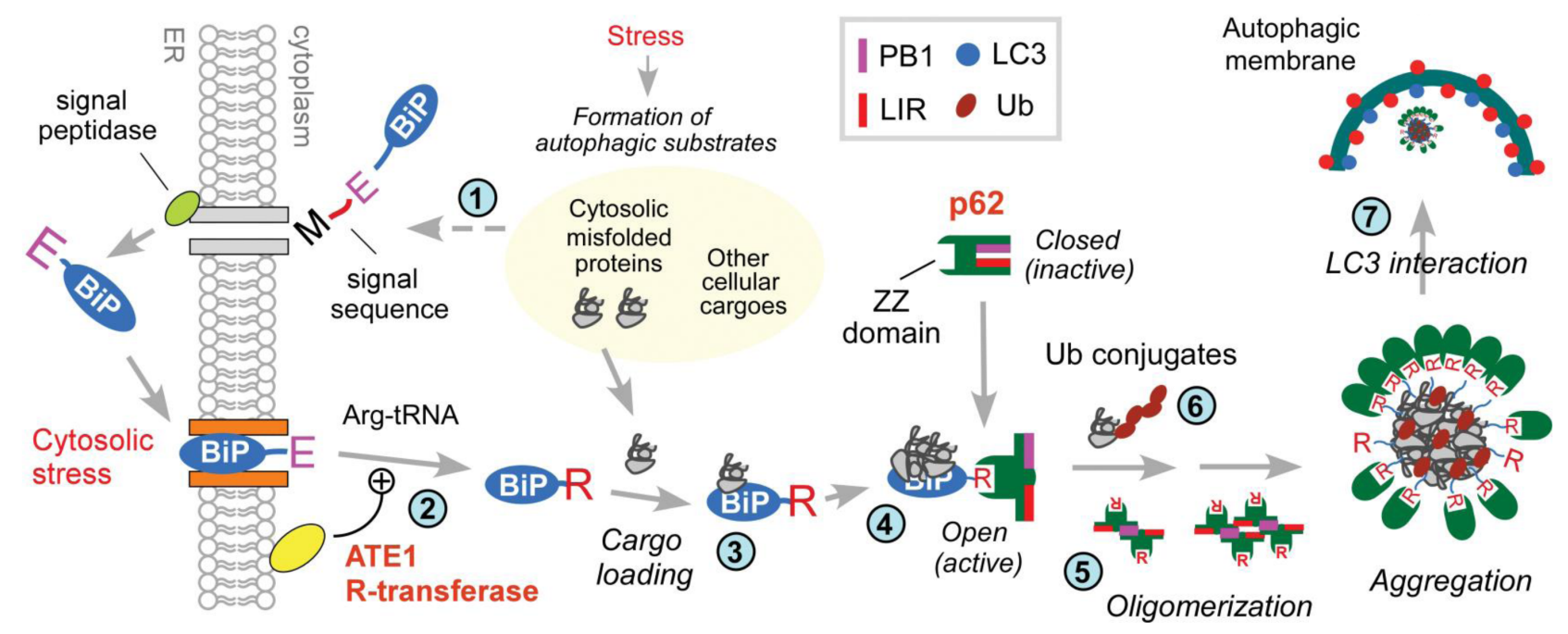

Fig. 1. Stress-induced $\mathrm{N}$-end rule pathway regulation of autophagy.

p62 and ATG5-dependent autophagic degradation (Fig. 1). This novel pathway protects cells from proteotoxic stress and promotes cell survival.

Our bioinformatics analysis revealed that in addition to BiP, many other ER residents also have the potential to acquire arginylation permissive pro-N-degrons, such as Glu and Asp, after losing their amino-terminal signal peptide upon entry. Indeed, we found that disulfide isomerase (PDI) and calreticulin (CRT) can also be arginylated under the same conditions that arginylate BiP. These results indicate that amino-terminal arginylation may behave like ubiquitinylation, in that it tags many proteins destined for destruction to increase the binding affinity of the substrates for $\mathrm{p} 62$.

\section{ACKNOWLEDGEMENTS}

This work was supported by the World Class Institute (WCI) Program (WCI 2009-002 to B.Y.K.), the Bio and Medical Technology Development Program (NRF-2014M3A9B5073938 to B.Y.K.), the Basic Science Research Program (NRF2013R1A2A2A01014170 to Y.T.K.) of the National Research Foundation (NRF) funded by the Ministry of Science, ICT and Future Planning (MSIP) of Korea, and also by the KRIBB Research Initiative Program (to B.Y.K) and NIH grant HL083365 (to Y.T.K). 